\title{
Nonexistence of Quantum Fields Associated with Two-Dimensional Spacelike Planes
}

\author{
Wulf Driessler and Stephen J. Summers \\ Fachbereich Physik, Universität Osnabrück, D-4500 Osnabrück, Federal Republic of Germany
}

\begin{abstract}
It is shown that in a relativistic quantum field theory satisfying Wightman's axioms, there are no nontrivial field-like operators, or even bilinear forms, associated to a two (or less)-dimensional spacelike plane in Minkowski space. This generalizes Wightman's result that fields can not be defined as operators at a point and stands in contrast to Borchers' result that field operators can be associated with one-dimensional timelike planes.
\end{abstract}

1. In this note we use analyticity properties associated with the Lorentz boosts in wedgelike spacetime regions (arising from Bisognano and Wichmann's identification of the modular automorphism group of wedge algebras with such boosts $[1,2])$ to prove that the polynomial operator algebras generated by the fields associated with a wedge and its causal complement are "irreducible" in a quantum field theory satisfying the Wightman axioms [3], so that, in a strong sense to be specified below, no nontrivial (unbounded) operator (or even bilinear form) on the physical Hilbert space can be associated with a two (or less)-dimensional spacelike plane. This latter point is a generalization of a result of Wightman [4] on the nonexistence of nontrivial field operators at a point, and is to be seen in contradistinction to a result of Borchers [5], that field operators can be associated with one-dimensional timelike planes. There are, of course, examples of fields at sharp times, i.e. associated with three-dimensional spacelike planes, e.g. the free scalar field.

2. To begin, we establish the framework and notation necessary for the statement and proof of the results. We assume the usual Wightman axioms, including Poincaré covariance, locality, spectrum condition and the uniqueness of the vacuum $\Omega$ (see [3] for further details), and we admit Bose and Fermi statistics. Let $W_{R}$ and $W_{L}$ denote the right and left wedges defined by

$$
W_{R}=\left\{x \in \mathbb{R}^{4}\left|x^{1}>\right| x^{0} \mid\right\}, \quad W_{L}=\left\{x \in \mathbb{R}^{4}\left|x^{1}<-\right| x^{0} \mid\right\},
$$

where $x^{0}$ is the time coordinate. Then $\mathscr{P}\left(W_{R}\right)$, respectively $\mathscr{P}\left(W_{L}\right)$, will signify the *-algebra consisting of all polynomials of field operators tested with functions from $\mathscr{S}\left(\mathbb{R}^{4}\right)$ that have support in $W_{R}$, respectively $W_{L}$. These operators are defined on 
a common, invariant, dense domain $D$ in the Hilbert space $\mathscr{H}$. Let $v\left(\hat{e}_{1}, t\right), t \in \mathbb{R}$, denote the one-parameter group of boost transformations leaving $W_{R}$ and $W_{L}$ invariant. Here $U(t)=U\left(v\left(\hat{e}_{1}, t\right)\right), t \in \mathbb{R}$, represents the associated strongly continuous group of unitary operators on $\mathscr{H}$, and $K_{1}$ its (unbounded) selfadjoint generator, i.e. $U(t)=e^{-i t K_{1}}$. In order to concisely express the locality properties (since we admit Fermi fields), it is customary to introduce a unitary operator $Z=\left(I+i U_{0}\right) /(1+i)$, where $U_{0}$ is the unitary operator on $\mathscr{H}$ representing a rotation of $2 \pi$ about any axis. Then one has

$$
[A, B] \psi=0, \forall \psi \in D, \forall A \in \mathscr{P}\left(W_{R}\right), \forall B \in Z \mathscr{P}\left(W_{L}\right) Z^{*} \equiv \mathscr{P}\left(W_{L}\right)^{z}
$$

Bisognano and Wichmann have shown under the above-mentioned assumptions (see Theorem 1 in [2]) that if $\Theta_{0}$ is the antiunitary TCP operator and $J$ is the antiunitary involution defined by $J=Z U_{\pi} \Theta_{0}$, where $U_{\pi}$ is the unitary operator corresponding to a (spatial) rotation of $\pi$ around the 1-axis, then one has

$$
J \mathscr{P}\left(W_{R}\right) J=\mathscr{P}\left(W_{L}\right)^{z}
$$

and the (dense) set $\mathscr{P}\left(W_{R}\right) \Omega$, respectively $\mathscr{P}\left(W_{L}\right)^{z} \Omega$, is a core for the (unbounded) selfadjoint operator $e^{\pi K_{1}}$, respectively $e^{-\pi K_{1}}$. Moreover, they have shown that

$$
\begin{aligned}
e^{\pi K_{1}} A \Omega=J A^{*} \Omega, & A \in \mathscr{P}\left(W_{R}\right), \\
e^{-\pi K_{1}} B \Omega=J B^{*} \Omega, & B \in \mathscr{P}\left(W_{L}\right)^{z} .
\end{aligned}
$$

We shall be interested in elements of $\mathscr{P}\left(W_{R}\right)$ and $\mathscr{P}\left(W_{L}\right)^{z}$ that are entire analytic with respect to $K_{1}$. By Lorentz covariance, one has $U(t) \varphi[f] U(t)^{-1}=\varphi\left[f_{t}\right]$, on $D$ (to minimize unnecessary notational complications, we pretend for the moment that all fields transform like the scalar Bose field; details for other spins are left to the reader), where $f_{t}(x)=f\left(v\left(\hat{e}_{1}, t\right) x\right)$. Since $W_{R}$ and $W_{L}$ are invariant under $v\left(\hat{e}_{1}, t\right) \forall t \in \mathbb{R}$, it is clear that $\mathscr{P}\left(W_{R}\right)$ and $\mathscr{P}\left(W_{L}\right)^{z}$ are invariant under conjugation by $U(t)$. Let now $\mathscr{P}_{e}\left(W_{R}\right)$ denote the set of all $A \in \mathscr{P}\left(W_{R}\right)$ for which $U(t) A U(t)^{-1}$, on $D$, is the restriction to the real axis of an entire analytic family of operators $A(\lambda) \in \mathscr{P}\left(W_{R}\right), \forall \lambda \in \mathbb{C}$, i.e. $\forall \psi \in D, A(\lambda) \psi$ is an entire analytic vector-valued function with restriction to the real axis $U(t) A U(t)^{-1} \psi$. Then for any $A \in \mathscr{P}_{e}\left(W_{R}\right)$,

$$
e^{-i \lambda K_{1}} A \Omega=A(\lambda) \Omega, \quad \forall \lambda \in \mathbb{C} .
$$

$\mathscr{P}_{e}\left(W_{L}\right)^{z}$ is defined similarly, and one has (4) for any $A \in \mathscr{P}_{e}\left(W_{L}\right)^{z}$, as well. We note that $\mathscr{P}_{e}\left(W_{R}\right) \Omega=\mathscr{P}_{e}\left(W_{L}\right)^{z} \Omega$, which can be seen as follows. For any $A \in \mathscr{P}_{e}\left(W_{R}\right), A \Omega=e^{\pi K_{1}} A(-i \pi) \Omega=J A^{*}(i \pi) \Omega$, using (4) and (3). By (2), $J A^{*}(i \pi) J \in \mathscr{P}\left(W_{L}\right)^{z}$, and since $J U(t)=U(t) J$, one sees easily that $J A^{*}(i \pi) J$ is an entire analytic element. This establishes the containment $\subset$; the opposite containment follows mutatis mutandis. We then have

$$
\mathscr{P}_{e}\left(W_{R}\right) \mathscr{P}_{e}\left(W_{L}\right)^{z} \Omega=\mathscr{P}_{e}\left(W_{L}\right)^{z} \mathscr{P}_{e}\left(W_{R}\right) \Omega=\mathscr{P}_{e}\left(W_{R}\right) \Omega .
$$

We next wish to show briefly that $\mathscr{P}_{e}\left(W_{R}\right) \Omega$ and $\mathscr{P}_{e}\left(W_{L}\right)^{z} \Omega$ are dense in $\mathscr{H}$. Let $g_{\rho}(t)=(\rho / \pi)^{1 / 2} e^{-\rho t^{2}}, \rho>0$, so that on $D, \int U(t) \varphi[f] U(t)^{-1} g_{\rho}(t) d t=\varphi\left[f_{\rho}\right]$, where $f_{\rho}(x)=\int f_{t}(x) g_{\rho}(t) d t \in \mathscr{P}\left(\mathbb{R}^{4}\right)$, as is easily seen. $\varphi[f] \in \mathscr{P}\left(W_{R}\right)$ implies $\varphi\left[f_{\rho}\right] \in \mathscr{P}\left(W_{R}\right), \forall 0<\rho<\infty$, and $U(t) \varphi\left[f_{\rho}\right] U(t)^{-1}$ has an extension to an entire 
analytic family of operators in $\mathscr{P}\left(W_{R}\right)$ given by $\varphi\left[(\rho / \pi)^{1 / 2} \int f_{s} \exp \left\{-\rho\left(s^{2}-2 s \lambda\right.\right.\right.$ $\left.\left.\left.+\lambda^{2}\right)\right\} d s\right], \lambda \in \mathbb{C}$. Since $g_{\rho}(t) d t \rightarrow \delta(t) d t$ weakly as measures as $\rho \rightarrow \infty$, and since, as $t \rightarrow 0,\left\|\left(\varphi\left[f_{t}\right]-\varphi[f]\right) \psi\right\| \rightarrow 0$ for any $f \in \mathscr{S}\left(\mathbb{R}^{4}\right)$ and $\psi \in D$, the claim follows.

3. We can now come to the core of our argument.

Theorem 1. Let $C$ be a bilinear form on $\mathscr{E} \times \mathscr{E}$, where $\mathscr{E}=\mathscr{P}_{e}\left(W_{R}\right) \Omega$, such that $\|C \Omega\|<\infty$ and $\left\|C^{*} \Omega\right\|<\infty$. Then $\langle A \phi, C \psi\rangle=\left\langle\phi, C A^{*} \psi\right\rangle, \forall \phi, \psi \in \mathscr{E}$ and $\forall A \in \mathscr{P}_{e}\left(W_{R}\right) \cup \mathscr{P}_{e}\left(W_{L}\right)^{z}$, implies $C=c I$, for some $c \in \mathbb{C}$.

Proof. A bilinear form $X$ can be decomposed into symmetric and antisymmetric parts $\left(X=X_{s}+X_{a}\right.$, where $\left.X_{s(a)}=\left(X+(-) X^{*}\right) / 2\right)$ and into $J$-invariant and -antiinvariant parts $\left(X=X_{i}+X_{a i}, X_{i(a i)}=(X+(-) J X J) / 2\right)$. Then $C$ can be decomposed uniquely into four pieces $C=C_{s, i}+C_{s, a i}+C_{a, i}+C_{a, a i}$, using the obvious notation. Since $\mathscr{P}_{e}\left(W_{R}\right) \cup \mathscr{P}_{e}\left(W_{L}\right)^{z}$ is invariant under the ${ }^{*}$-operation and under conjugation by $J$ (by (2) and $J U(\lambda) J=U(\bar{\lambda})[2]$ ), if $C$ satisfies the hypothesis, then so does each of the elements of this decomposition. We consider first $C_{s, i}$.

Let $A \in \mathscr{P}_{e}\left(W_{R}\right)$ and $B \in \mathscr{P}_{e}\left(W_{L}\right)^{z}$ be symmetric. Then, by assumption, for any $\lambda, \lambda^{\prime} \in \mathbb{C}$

$$
\left\langle A(\bar{\lambda}) \Omega, C_{s, i} B\left(\lambda^{\prime}\right) \Omega\right\rangle=\left\langle\overline{A(\lambda) B\left(\lambda^{\prime}\right) \Omega, C_{s, i}^{*} \Omega}\right\rangle=\left\langle B\left(\overline{\lambda^{\prime}}\right) \Omega, C_{s, i} A(\lambda) \Omega\right\rangle,
$$

which we shall call $F_{s, i}\left(\lambda, \lambda^{\prime}\right)$. Since $C_{s, i}^{*} \Omega \in \mathscr{H}$ and $A$ and $B$ are entire analytic elements, (6) is separately entire analytic in $\lambda$ and $\lambda^{\prime}$, and therefore, by Hartogs' theorem, jointly entire. From (4) and (6) we conclude

$$
F_{s, i}\left(\lambda, \lambda^{\prime}\right)=\left\langle e^{-i \bar{\lambda}^{\prime} K_{1}} B \Omega, C_{s, i} e^{-i \lambda K_{1}} A \Omega\right\rangle, \forall \lambda, \lambda^{\prime} \in \mathbb{C} .
$$

Restriction to the diagonal $\lambda=\lambda^{\prime}$ defines a bounded holomorphic function $f_{s, i}(\lambda)$ in $\mathbb{R} \times[0, \pi]$, and by (7) the restriction to $\operatorname{Im} \lambda=\pi$ is equal to

$$
\begin{aligned}
f_{s, i}(t+i \pi) & =\left\langle e^{-\pi K_{1}} B(t) \Omega, C_{s, i} e^{\pi K_{1}} A(t) \Omega\right\rangle \\
& =\left\langle J B(t) \Omega, C_{s, i} J A(t) \Omega\right\rangle=\left\langle\overline{B(t) \Omega, C_{s, i} A(t) \Omega}\right\rangle=f_{s, i}(t),
\end{aligned}
$$

using (3) and the fact that $C_{s, i}$ is $J$-invariant and symmetric. We may thus extend $f_{s, i}(\lambda)$ to a bounded, periodic, entire function, which by Liouville's theorem must be a constant. Thus, the restriction $f_{s, i}(t)$ is independent of $t$, and since $w$ - $\lim U(t)=P_{\Omega}$, the projection onto the vacuum [6], we have

$$
\left\langle\overline{A(t) B(t) \Omega, C_{s, i} \Omega}\right\rangle=\langle\Omega, A B \Omega\rangle\left\langle\overline{\Omega, C_{s, i} \Omega}\right\rangle, \forall t \in \mathbb{R},
$$

using (6) and $\left\|C_{s, i} \Omega\right\|<\infty$.

Up to (8) the calculation is independent of the particular choice $C_{s, i}$. And in (8) a simple calculation yields the same equality for $C_{a, a i}$ and $f(t+i \pi)=-f(t)$ for $C_{s, a i}$ and $C_{a, i}$. We conclude that (9) holds for general $C$ satisfying the hypothesis of the theorem. Taking $A=I$, we have for any such $C$

$$
\overline{\left\langle\left(B_{1}+i B_{2}\right) \Omega, C \Omega\right\rangle}=\left\langle\Omega,\left(B_{1}+i B_{2}\right) \Omega\right\rangle\langle\overline{\Omega, C \Omega}\rangle,
$$

for all symmetric $B_{1}$ and $B_{2}$ in $\mathscr{P}_{e}\left(W_{L}\right)^{z}$, so that $C \Omega \perp B \Omega$ for all $B \in \mathscr{P}_{e}\left(W_{L}\right)^{z}$, such 
that $B \Omega \perp \Omega$. Thus, $C \Omega=c \Omega$, for some $c \in \mathbb{C}$, which implies

$$
\langle A \Omega, C B \Omega\rangle=\left\langle B^{*} A \Omega, C \Omega\right\rangle=c\left\langle B^{*} A \Omega, \Omega\right\rangle=c\langle A \Omega, B \Omega\rangle,
$$

$\forall A \in \mathscr{P}_{e}\left(W_{R}\right), B \in \mathscr{P}_{e}\left(W_{L}\right)^{z}$. The conclusion then follows.

Remark. The assumption $\|C \Omega\|<\infty$ may look unnecessarily strong at first glance. However, consider a free scalar field $\varphi$ and define $\varphi(0)$ as a form limit of $\left\langle\psi, \varphi\left(g_{\rho}\right) \phi\right\rangle$ for $\psi, \phi \in D$ and $\left\{g_{\rho}\right\}$ converging to a $\delta$-function. Then $\langle U(t) \psi, \varphi(0) U(t) \phi\rangle$ is indeed independent of $t$, but $\langle\Omega, \varphi(0) \Omega\rangle=0$, and the form is certainly not identically zero.

4. Theorem 1 indicates that the set of operators $\mathscr{P}\left(W_{R}\right) \cup \mathscr{P}\left(W_{L}\right)^{z}$ is in a very strong sense irreducible, as is, therefore, $\mathscr{P}\left(\gamma W_{R}\right) \cup \mathscr{P}\left(\gamma W_{L}\right)^{z}$, for any element $\gamma$ of the restricted Poincaré group $\mathscr{P}_{+}^{\uparrow}$, where $\gamma W$ signifies the image of the region $W$ under the map on Minkowski space represented by $\gamma$. The intersection $\mathscr{I}=\overline{W_{R}} \cap \overline{W_{L}}$ is the two-dimensional spacelike plane $\left\{x \in \mathbb{R}^{4} \mid x^{0}=x^{1}=0\right\}$, and the set $\left\{\gamma \mathscr{I} \mid \gamma \in \mathscr{P}{ }_{+}^{\dagger}\right\}$ contains all two-dimensional spacelike planes in Minkowski space. The points in $\gamma \mathscr{I}$ are all spacelike separated from those in $\gamma W_{R} \cup \gamma W_{L}$, so that in local quantum field theory one would expect field objects to commute (or anticommute) with those in $\mathscr{P}\left(\gamma W_{R}\right) \cup \mathscr{P}\left(\gamma W_{L}\right)$. Theorem 1 would then entail their triviality. We shall now state and prove a strong form of this intuition.

Given any open set $\mathcal{O} \subset \mathbb{R}^{4}$ containing a set $K=\gamma_{0} \mathscr{I}, \gamma_{0} \in \mathscr{P}{ }_{+}^{\uparrow}$, let $\mathscr{2}(\mathcal{O})$ denote the set of all operators $C$ defined, along with its adjoint, on the domain $U\left(\gamma_{0}\right) \mathscr{E}$ satisfying

$$
\langle A \phi, C \psi\rangle=\left\langle C^{*} \phi, A^{*} \psi\right\rangle, \forall A \in \mathscr{P}\left(\mathcal{O}^{\prime}\right)^{z}, \forall \phi, \psi \in U\left(\gamma_{0}\right) \mathscr{E},
$$

where $\mathcal{O}^{\prime}$ denotes the interior of the causal complement of $\mathcal{O}$ and $\mathscr{P}\left(\mathcal{O}^{\prime}\right)$ is the polynomial algebra generated by the field operators with support in $\mathcal{O}^{\prime}$. Clearly $\mathscr{P}(\mathcal{O})$ $\subset \mathscr{Q}(\mathcal{O})$. Let further $\overline{\mathscr{2}(\mathcal{O})}$ denote the closure of $\mathscr{2}(\mathcal{O})$ in a topology chosen so that each element in $\overline{\mathscr{Q}(\mathcal{O})}$ satisfies $(10)$ and $\|Q \Omega\|<\infty,\left\|Q^{*} \Omega\right\|<\infty, \forall Q \in \overline{\mathscr{Q}(\mathcal{O})}$. It is clear that topologies weaker than the Wightman topology may be admitted. Let, furthermore, $\mathscr{L}(K)$ denote the set of all open $\mathcal{O} \subset \mathbb{R}^{4}$ containing $K=\gamma_{0} \mathscr{I}$ such that $\mathcal{O}^{\prime}=\gamma_{1} W_{R} \cup \gamma_{2} W_{L}$, for some $\gamma_{1}, \gamma_{2} \in \mathscr{P}_{+}^{\uparrow}$.

Corollary 1. For any $K=\gamma_{0} \mathscr{I}, \gamma_{0} \in \mathscr{P P}_{+}^{\uparrow}$, as above, $\bigcap_{\mathcal{O} \in \mathscr{L}(K)} \overline{\mathscr{Q}(\mathcal{O})}=\{\mathbb{C I}\}$.

Proof. Let $C \in \bigcap_{\mathcal{O} \in \mathscr{L}(K)} \overline{\mathscr{2}(\mathcal{O})}$. Then (10) holds for all $A \in \bigcup_{\mathcal{O} \in \mathscr{L}(K)} \mathscr{P}\left(\mathcal{O}^{\prime}\right)^{z}$. Since $\bigcup_{\mathcal{O} \in \mathscr{L}(K)} \mathcal{O}^{\prime}=$ $\gamma_{0} W_{R} \cup \gamma_{0} W_{L}$ and since the Poincaré transformations act continuously on $\mathscr{S}\left(\mathbb{R}^{4}\right)$, Wightman continuity entails that (10) holds in fact for all $A \in \mathscr{P}\left(\gamma_{0} W_{R}\right)^{z} \cup \mathscr{P}\left(\gamma_{0} W_{L}\right)^{z}$. For the sake of convenience, we rewrite this as:

$$
\langle A \phi, \tilde{C} \psi\rangle=\left\langle\phi, \widetilde{C} A^{*} \psi\right\rangle, \forall A \in \mathscr{P}_{e}\left(W_{R}\right)^{z} \cup \mathscr{P}_{e}\left(W_{L}\right)^{z}, \forall \phi, \psi \in \mathscr{E},
$$

where $\widetilde{C} \equiv U\left(\gamma_{0}\right)^{-1} C U\left(\gamma_{0}\right)$. Equation (11) clearly holds for $\tilde{C}^{*}$ and $U_{0} \tilde{C} U_{0}$, as well as $E \widetilde{C} E$, where $E=U_{\pi} \Theta_{0}$ (note $\mathscr{P}_{e}\left(W_{R}\right)^{z} \cup \mathscr{P}_{e}\left(W_{L}\right)^{z}$ is invariant under conjugation by $E$ ). Therefore, it holds for each of the elements in the decomposition of $C$ into pieces with \pm parity under each of these three operations, as do the domain 
requirements. To minimize notation we assume $\widetilde{C}$ invariant under conjugation by $E$; the proof of the other case shall be clear. Writing $2 X_{ \pm}=\left(X \pm U_{0} X U_{0}\right)$, and noting that $Z X_{-} Z^{*}=i U_{0} X_{-}=-i X_{-} U_{0}$, and $Z X_{+} Z^{*}=X_{+}$, we have for any $\phi, \psi \in \mathscr{E}$,

$$
\begin{aligned}
& \left\langle A \phi, \tilde{C}_{+} \psi\right\rangle=\left\langle\phi, \tilde{C}_{+} A^{*} \psi\right\rangle, \forall A \in \mathscr{P}_{e}\left(W_{R}\right) \cup \mathscr{P}_{e}\left(W_{L}\right)^{z}, \\
& \left\langle A \phi, \tilde{C}_{-} \psi\right\rangle=\left\langle\phi, \tilde{C}_{-} A^{*} \psi\right\rangle, \forall A \in \mathscr{P}_{e}\left(W_{R}\right)^{+} \cup \mathscr{P}_{e}\left(W_{L}\right)^{z}, \\
& \left\langle A \phi, \tilde{C}_{-} \psi\right\rangle=-\left\langle\phi, \tilde{C}_{-} A^{*} \psi\right\rangle, \forall A \in \mathscr{P}_{e}\left(W_{R}\right)^{-} \cup \mathscr{P}_{e}\left(W_{L}\right)^{-},
\end{aligned}
$$

where e.g. $\mathscr{P}_{e}\left(W_{R}\right)^{+}$denotes the set $\left\{X_{+} \mid X \in \mathscr{P}_{e}\left(W_{R}\right)\right\}$. Equation (12) and Theorem 1 yield at once the triviality of $\widetilde{C}_{+}$. We show that (13) and (14), along with trivial modifications in the proof of Theorem 1, yield the triviality of $\widetilde{C}_{-}$, therefore of $C$.

Let $A \in \mathscr{P}_{e}\left(W_{R}\right)$ and $B \in \mathscr{P}_{e}\left(W_{L}\right)^{z}$ be symmetric and decompose them into their even and odd parts under $U_{0}$, which remain symmetric and entire analytic. By (13) one has

$$
\begin{aligned}
F_{s}\left(\lambda, \lambda^{\prime}\right) & =\left\langle\left(A_{+}(\bar{\lambda})+A_{-}(\bar{\lambda})\right) \Omega, \widetilde{C}_{-, s}\left(B_{+}\left(\lambda^{\prime}\right)+B_{-}\left(\lambda^{\prime}\right)\right) \Omega\right\rangle \\
& =\left\langle\left(B_{+}\left(\overline{\lambda^{\prime}}\right)+B_{-}\left(\overline{\lambda^{\prime}}\right)\right)\left(A_{+}(\bar{\lambda})+A_{-}(\bar{\lambda})\right) \Omega, \tilde{C}_{-, s} \Omega\right\rangle .
\end{aligned}
$$

Since $\left\|\tilde{C}_{-, s} \Omega\right\|<\infty, F_{s}\left(\lambda, \lambda^{\prime}\right)$ is an entire analytic function in $\mathbb{C}^{2}$. Restricting to the diagonal $\lambda=\lambda^{\prime}$ in $(\mathbb{R} \times[-\pi, 0])^{2}$, and taking the restriction to $\operatorname{Im} \lambda=-\pi$, we have from (3)

$$
f_{s}(t-i \pi)=\left\langle J\left(A_{+}(t)+A_{-}(t)\right) \Omega, \tilde{C}_{-, s} J\left(B_{+}(t)+B_{-}(t)\right) \Omega\right\rangle .
$$

Since $J<Z E$ and $\tilde{C}_{-, s}$ is invariant under conjugation by $E$ (since $\widetilde{C}$ is, and all the operations commute), we have $J \widetilde{C}_{-, s} J=Z \tilde{C}_{-, s} Z^{*}=i U_{0} \tilde{C}_{-, s}$. Therefore,

$$
\begin{aligned}
f_{s}(t-i \pi) & =\left\langle\overline{\left(A_{+}(t)+A_{-}(t)\right) \Omega, i U_{0} \tilde{C}_{-, s}\left(B_{+}(t)+B_{-}(t)\right) \Omega}\right\rangle \\
& =-i\left\langle\overline{\left(A_{+}(t)-A_{-}(t)\right) \Omega, \tilde{C}_{-, s}\left(B_{+}(t)+B_{-}(t)\right) \Omega}\right\rangle \\
& =-i\left\langle\left(A_{+}(t)+A_{-}(t)\right) \Omega, \tilde{C}_{-, s}\left(B_{+}(t)+B_{-}(t)\right) \Omega\right\rangle,
\end{aligned}
$$

which equals $-\mathrm{if}_{s}(t)$, where we have used (13) and (14). Thus, we can apply the argument of Theorem 1 to $f_{s}(\lambda)^{4}$ to show $f_{s}(t)$ independent of $t$. A similar calculation yields the same result for each of the elements in $\widetilde{C}$ 's decomposition, and the argument presented earlier completes the proof.

The same triviality result would follow for any region $K$ in $\mathbb{R}^{4}$ that can be embedded in a two-dimensional spacelike plane.

5. Let us finally remark that completely analogous results can be obtained if the field algebras $\mathscr{P}(W)$ are replaced by von Neumann algebras $\mathfrak{R}(W)$ in a Haag-Araki theory of local observables supplemented by the requirement that the modular automorphism group of $\mathfrak{R}(W)$ equals $U(t), t \in \mathbb{R}$. Such algebras can be obtained from a Wightman field theory satisfying an additional assumption (see $[1,2])$. The nonexistence of bounded or unbounded field objects on a two-dimensional spacelike plane partly extends results of [7], where only sets $K$ with compact closure and bounded operators are considered. (See also [8] for another treatment of certain $K$ with compact closure.) We point out, however, that as long as one can associate operators or forms with the local structure in the sense that, for example, there is a 
sequence $P_{n} \in \mathscr{P}_{s}\left(\mathcal{O}_{n}\right)$, with $\mathcal{O}_{n}$ the causal complement of $W_{L, n} \cup W_{R}$ and $W_{L, n}$ an increasing sequence of left wedges with $\bigcup_{n} W_{L, n}=W_{L}$, such that $s$-lim $P_{n} \Omega=\phi-$ which defines a form in a natural way - then triviality of this form can also be shown by using the argument in the proof of Theorem 2.2 in [9]. The virtue of the proof presented here is precisely that the operator or form in question need not be associated with the local structure in this sense.

\section{References}

1. Bisognano, J. J., Wichmann, E.H.: On the duality condition for a Hermitian scalar field. J. Math. Phys. 16, 985 (1975)

2. Bisognano, J.J., Wichmann, E. H.: On the duality condition for quantum fields. J. Math. Phys. 17, 303 (1976)

3. Streater, R. F., Wightman, A. S.: PCT, spin and statistics, and all that. New York: Benjamin 1964

4. Wightman, A. S.: La théorie quantique locale et la théorie quantique des champs. Ann. Inst. H. Poincaré 1, 403 (1964)

5. Borchers, H. J.: Field operators as $C^{\infty}$ functions in spacelike directions. Nuovo Cimento 33, 1600 (1964)

6. Driessler, W.: On the structure of fields and algebras on null planes I. Local algebras. Acta Phys. Austriaca 46, 63 (1977)

7. Woronowicz, S.: A generalization of a theorem by Wightman. Commun. Math. Phys. 9, 142 (1968)

8. Napiórkowski, K., Woronowicz, S.: Bull. Acad. Pol. Sci. 14, 265 (1966)

9. Driessler, W.: Comments on lightlike translations and applications in relativistic quantum field theory. Commun. Math. Phys. 44, 133 (1975)

Communicated by K. Osterwalder

Received November 3, 1982 